\title{
MEANDERING THOUGHTS: AFIR AND THE MILLENNIUM
}

The well-publicised millennium bug is very much on my mind these days. As head of information technology at a large investment bank, I am ultimately responsible for the successful remediation of all our critical computer systems by the time the third millennium dawns in the Christian calendar. The millennium bug poses a fascinating risk theory problem, although not of the kind that is generally analysed and written about in the ASTIN Bulletin. The legal profession has entered the year 2000 picture, hoping to fashion a short-term, but highly lucrative, practice from the various liability suits that will certainly be filed in connection with failures of computer systems afflicted with the millennium bug. A few writers of commercial liability insurance have devised and offered covers to deal with this special type of legal risk.

There are several interesting mathematical aspects of the year 2000 problem. Many financial services firms have written a large amount of proprietary software that is critical to the successful functioning of those firms each day. Most of this software is afflicted with the millennium bug, and thus needs to be remediated. An information technology project to deal with the year 2000 problem for proprietary software begins with an inventory of all critical systems. Each system in the inventory must be assessed as to year 2000 compliance. This involves inspecting each line of computer code to find offending date references and then fixing those bugs. Several different tools have been developed to search for and locate such bugs automatically. The trouble is that assessment tools are not perfect. The most insidious imperfection is a "false negative", indicating incorrectly that a millennium bug has not been detected.

The occurrence of false negatives is generally discovered during the unit-testing phase of a firm's year 2000 compliance program. Unit testing involves putting each critical computer system through a time-warp test in which the clock ticks over from December 31st, 1999 to January 1st, 2000 (or January 3rd, 2000 in the U.S. and January 4th, 2000 elsewhere in the world, the first business days of the new millennium) to verify that the computer system has been remediated properly. If the system fails, it can be due to improper and inadequate remediation or to the existence of a false negative. If the system fails due to a false negative, the assessment tool must be modified to search for the new type of bug, and the whole process of assessment, remediation, and unit testing must begin anew. This iterative process is generally terminated at some point of diminishing returns, and statistical analysis of the testing results can be used to determine such a point. 
The risk theory aspects of the year 2000 problem lie in considering the population of systems within a given firm, and then the population of firms and other entities that deal with one another. Many systems within a firm are interdependent. A problem in one can bring down others, inducing a cascade effect. The same is true among firms. Firms deal with one another and with various regulatory agencies and industry bodies. Firms are dependent on vendors of software. Firms transact with clients who have to remediate their own systems. This network of dependencies extends beyond domestic borders to the whole world.

Such critical system and network dependencies are not limited to financial institutions. All firms have computer systems. Any firm must have electric power and telecommunications systems to operate. The lifts and various other parts of building infrastructure must work properly. In short, there are many things that can go wrong, and there is a high degree of contagion due to interconnectedness. How many problems need to arise within the "network of computer systems and firms" before a given firm ceases to operate properly? How long would such a condition have to exist before the firm was ruined financially? These are difficult questions to answer. With their solid background in mathematics and risk, actuaries should have something useful to say about this problem. Yet, I am unaware of any seminal actuarial papers on this subject.

What is the connection between AFIR and the millennium? It could be about actuaries writing papers on the risks of the millennium bug. However, as the current chairperson of AFIR, the millennium is on my mind in another way. I have just returned from the successful 8th AFIR colloquium that was held at Cambridge University in England from September 14th to September 17th, 1998. When reviewing the terms of office for the various members of the AFIR Council, I realized that my own term expires in the year 2000. I began to consider in what state of affairs I would like to see AFIR on the presumably momentous occasion of this 10 th colloquium to be held in Tromso, Norway in June 2000. And I began to realise that just as a networked society is key to understanding the millennium bug, a networked society is also key to understanding the potential of AFIR.

The primary purpose of AFIR is to bring together actuaries and nonactuaries, academics and practitioners, students and non-students, who are interested in the measurement and management of financial and investment risks. AFIR is required to hold an annual colloquium, except in any year in which an International Congress of Actuaries (ICA) is held. The early colloquia were so successful that the AFIR Council decided to hold a colloquium in 1995 when there was an ICA in Brussels, Belgium and in 1998 when there was an ICA in Birmingham, England. The Council is entertaining a serious proposal for a colloquium in 2002, also an ICA year. Globally there are currently about 2,000 (coincidentally!) members of AFIR. Generally only $5 \%$ to $15 \%$ of these members attend an annual colloquium. So AFIR has a challenge in staying connected with its full membership. 
At its recent meeting in Cambridge, England, the AFIR Council discussed how the internet could be used to help meet the challenge of keeping AFIR members connected and engaged between AFIR colloquia. The newly-reorganized International Association of Actuaries (IAA), which is the parent of the AFIR Section, has introduced a web site with internet address www.actuaries.org. After selecting either the English or Français button on the initial home page, one arrives at a more complete home page with three rows of buttons at the top. Near the middle of the second row is a button marked AFIR that takes the visitor to the AFIR home page.

A visitor to the AFIR home page can easily send an e-mail to any Council member who has an internet address. The locations and dates of future AFIR Colloquia are also shown on the page. Finally, there is brief description of AFIR itself. At the present time, one could hardly claim that the content of the AFIR home page is very rich. However, it is easy to imagine how we could make it of much greater utility to AFIR members and non-members by providing access to a catalogue of authors, titles, and abstracts of papers presented at past AFIR colloquia. The list would also provide an e-mail address and/or phone number for each author. Ultimately, we might find an easy-to-maintain method to provide direct access to the papers themselves, without having to force the AFIR home page visitor to contact the authors directly.

Arnaud Clement-Grandcourt, the current Vice-Chairperson of AFIR, has suggested providing access to other research from the AFIR home page. In particular, he cited the research currently being conducted by a working group of actuaries in France on the relationship between risk measurement and performance attribution for mutual funds. The Council has asked Arnaud to explore vigorously the internet possibilities for AFIR in its quest to attract members and to help them network better with each other. As we head into the third millennium, we feel that we must harness the rapidly growing power and reach of information technology to strengthen AFIR's social network. Please send me your suggestions as to how AFIR can achieve this goal. Visit the AFIR home page and send me an e-mail directly from there.

JAMES A. TILLEY

Chairperson, AFIR 